\title{
EDITORIAL Genomic imprinting: theories and data
}

\author{
Heredity (2014) 113, 93-95; doi:10.1038/hdy.2014.52
}

In his inaugural professorial lecture at Cambridge, William Bateson, one of the fathers of Mendelian genetics, admonished students of the new science:

If I may throw out a word of counsel to beginners, it is: Treasure your exceptions! ... Keep them always uncovered and in sight. Exceptions are like the rough brickwork of a growing building which tells that there is more to come and shows where the next construction is to be. (Bateson, 1908)

This advice has borne much fruit in the intervening century, leading to the discovery of numerous fundamental aspects of genetics, including linkage, gene interactions and sex chromosomes. In the last two decades, we have begun to uncover a particularly rich source of such Batesonian exceptions to standard Mendelian expression resulting from forms of epigenetic inheritance such as genomic imprinting.

Throughout much of the twentieth century, it was assumed that for autosomal genes, paternal and maternal contributions to diploid offspring were identical and so the genotypically identical reciprocal heterozygotes would necessarily manifest the same phenotypes. The first exceptions to this rule were noted in the peculiar behavior of some dipteran chromosomes (Metz, 1938); much later, the expression of transgenes in mice was also found to contradict the usual rules of expression (Reik et al., 1987; Sapienza et al., 1987). Neither of these phenomena would now be viewed by most geneticists as genomic imprinting: the term is now normally used to refer to the differential expression of endogenous genes according to their parental origin. The first mammalian gene shown to be imprinted was the mouse insulin-like growth-factor 2 receptor (Igf2r; Barlow et al., 1991), with unequivocal examples of imprinting (in the sense of the modern concept) so far found only in mammals and angiosperms. The archetypal example is Igf-2, which in all therian mammals examined to date is expressed in most (but not all) fetal tissues solely from the paternal copy (Smits et al., 2008).

It is clear that the number of imprinted genes is small, no moreand possibly considerably less - than a few hundred (Kelsey and Bartolomei, 2012). Nevertheless, the research springing from these rare exceptions has led to significant advances in our understanding of gene expression and evolution. For example, the burgeoning field of epigenetics has benefitted greatly from work done to discover how DNA methylation affects expression at imprinted loci (FergusonSmith, 2011). The theoretical hypotheses involving the role of genetic conflict in the evolution of imprinting have contributed to the realization that genome evolution in general is subject to a variety of conflicting selection pressures (Lachmann et al., 2003). Even medicine has profited, with the discovery that errors in imprinting status can lead to growth disorders and cancer (reviewed in Lee and Bartolomei, 2013).
In spite of this progress, however, it has become increasingly clear that the interactions between evolutionary theorists and molecular empiricists interested in genomic imprinting could be improved. Evolutionary theorists depend on the molecular empiricists to provide the biological grounding that allows them to derive realistic models, whereas molecular empiricists rely on the theorists to provide predictions and interpretations of patterns grounded in evolution. Yet at the same time as theorists have struggled to keep abreast of numerous rapid technological and methodological advances and voluminous amounts of data, empiricists have largely ignored many of the recent theoretical innovations and debates. The meeting sponsored by the National Evolutionary Synthesis Center (NESCent) at Duke University, North Carolina (funded by the National Science Foundation, USA), in December 2011, was a step towards reintegrating the field. The goal was for the latest data to inform the theories and for empiricists to recognize ways their data could both test existing theories and to suggest new ones. Most of the papers in this special issue arose from ideas developed in discussions at this meeting, and we are most grateful to NESCent for the opportunity to be able to meet with their generous support.

Genomic imprinting has inspired considerable work in evolutionary theory, in part, because the use of just one copy of a gene when an organism has two requires some sort of evolutionary explanation. Similarly, the phylogenetic distribution of imprinting, as well as the chromosomal clustering of many (but not all) imprinted genes, both demand and suggest evolutionary hypotheses. The most prominent rationale for the evolution of imprinting is Haig and colleagues' genetic conflict or kinship hypothesis (Haig and Graham, 1991; Moore and Haig, 1991), which argues that imprinting is the result of a conflict of interest between paternally and maternally derived genes. In this issue, Haig (2014) points out that this hypothesis does not mean that parents and offspring (or mothers and fathers) are in conflict. Indeed, genetic conflict can lead to cooperation between mother and offspring, a crucial realization given that such cooperation has motivated a number of other hypotheses. The genetic conflict hypothesis has implications for our understanding of the 'units-ofselection debate, too, as Gardner (2014) shows here. In his paper he observes that, under this hypothesis, imprinting evolves as the result of selection among individuals and yet the interpretation is one of conflict among differently derived elements of that individual's genome. Gardner's contribution is a formal analysis of this apparent paradox.

The predominance of the genetic conflict hypothesis has not prevented the propounding of other theories for the evolution of imprinting, however. Spencer and Clark (2014) review some 13 of these 'non-conflict' evolutionary hypotheses, suggesting that several of them are tenable explanations that should be considered by both empiricists and theorists. They argue that imprinting may have arisen 
for different reasons at different loci in different groups of organisms. In a similar vein, Patten et al. (2014) compare and contrast three of the most prominent hypotheses, namely genetic conflict (Haig and Graham, 1991; Moore and Haig, 1991), sexual antagonism (Day and Bonduriansky, 2004) and maternal-offspring coadaptation (Wolf and Hager, 2006). They derive specific, discriminating predictions of these different ideas, which they hope will inspire empirical work aimed at testing these predictions.

Wolf and Brandvain (2014) also examine a subset of the long list of hypotheses for the evolution of imprinting. They focus on models holding that imprinting arose because it allows certain genetic factors to interact in a more coordinated way. For example, these genetic factors may be alleles at different loci within an individual that in combination confer high fitness on the bearer, or they may be genes for which matching expression in a parent and its offspring is beneficial. The authors contrast such models with those (for example, genetic conflict) that invoke imprinting's gene dosage effects as causative.

As noted above, the phylogenetic distribution of imprinting is clearly significant, with the apparent absence in monotremes pinpointing to its evolutionary origin in vertebrates to after the monotreme-therian split $\sim 170$ myr ago, coincident with the origin of the placenta. As Keverne (2014) argues in his paper, the properties of mammalian pregnancy are critical to the role played by imprinted genes. The coexistence of three female generations (mother, female fetus and the latter's oocytes) provides an opportunity for selection to coordinate gene expression to further maternal-fetal coadaptation through coexpression of the same female-derived alleles. He concludes that the existing machinery for imprinting (perhaps a form of genome defence) was coopted to this end.

The critically different reproductive biology of marsupials and eutherians, especially in the function of the placenta, has obvious implications for how and why imprinting evolved. The paper by Stringer et al. (2014) notes that imprinting has a critical role in eutherian pregnancy, with most imprinted genes expressed in the placenta and fetus. In marsupials, however, pregnancy is much shorter and the part of the eutherian placenta in supplying nutrition to the developing fetus is instead played by the mammary gland delivering milk to the altricial neonate. Hence, imprinting in marsupials is more likely to affect genes in the mammary gland involved in postnatal lactation rather than placentally active genes during pregnancy, and there is increasing evidence in favor of this expectation.

The discovery of imprinted genes has often been driven by technological and methodological advances (Ferguson-Smith, 2011). This progress has not been without hitches, however: see the criticisms of DeVeale et al. (2012) and Okae et al. (2012) of different aspects of the study of Gregg et al. (2010) that had suggested that $>1000$ mouse genes may be imprinted. In this issue, Wang and Clark (2014) discuss the technicalities surrounding the use of RNA sequencing of reciprocal crosses and show the power of this approach to discover imprinted genes in non-model species. Such data are essential if we are to understand just where in the mammalian phylogeny different genes became or ceased to be imprinted. Wang and Clark (2014) report that the frequency with which such changes in imprinting status occurred is surprisingly high, an observation that has important implications for many of the hypotheses for the evolution of imprinting.

By generating reciprocal heterozygotes, reciprocal crosses between species or between different inbred lines within a species have played a crucial role in discovering imprinted genes. But, as Wolf et al. (2014) note, such hybrids sometimes have unusual gene expression, even to the point of manifesting lethal incompatibilities. These authors review the evidence concerning imprinted gene expression in intra- and interspecific hybrids and examine the evolutionary scenarios under which imprinting could contribute to abnormal development in hybrids and its potential role in speciation.

Finally, Hanna and Kelsey (2014) argue that theories concerning the evolution of mammalian imprinting can benefit from recent molecular insights into the timing of the establishment and maintenance of the epigenetic marks (exemplified by methylation) at imprinting control regions. These marks are not the result of specific imprinting machinery in gametogenesis; instead they differ from other epigenetic modifications in their maintenance during the genome-wide demethylation early in development. In other words, the template of differential methylation established during gametogenesis is modified by embryonic events. The evolution of imprinting at previously unimprinted loci, therefore, is likely to depend on alterations in these latter processes.

This collection of papers clearly shows the rewards of heeding Bateson's advice and treasuring the defining exception to standard genetic expression that characterizes genomic imprinting. As a result of continuing research into this rare and once obscure phenomenon, we now know much more on subjects as diverse as molecular genetics (notably its critical intricacies during early development), natural selection (particularly the multifarious ways in which it can act on different genomic elements) and mammalian evolution (especially the consequences of placental evolution). Most pleasingly, these papers show that dialogue among the wide range of scientists interested in genomic imprinting can lead to genuine advances in many facets of biological enquiry.

\section{CONFLICT OF INTEREST}

The authors declare no conflict of interest.

HG Spencer ${ }^{1}$ and JB Wolf ${ }^{2}$
${ }^{1}$ Allan Wilson Centre and Gravida: National Centre for Growth and
Development, University of Otago, Dunedin, New Zealand and
${ }^{2}$ Department of Biology and Biochemistry, University of Bath, Bath, UK

E-mail: hamish.spencer@otago.ac.nz

Barlow DP, Stoger R, Herrmann BG, Saito K, Schweifer N (1991). The mouse insulin-like growth factor type-2 receptor is imprinted and closely linked to the Tme locus. Nature 349: 84-87.

Bateson W (1908). The method and scope of genetics. (Inaugural Lecture, 23 October 1908.) Reprinted in: (ed Bateson B) William Bateson, F.R.S.: Nasturalist. Cambridge University Press, Cambridge (1928) 317-333.

Day T, Bonduriansky R (2004). Intralocus sexual conflict can drive the evolution of genomic imprinting. Genetics 167: 1537-1546.

DeVeale B, van der Kooy D, Babak T (2012). Critical evaluation of imprinted gene expression by RNA-Seq: a new perspective. PLoS Genet 8: e1002600.

Ferguson-Smith AC (2011). Genomic imprinting: the emergence of an epigenetic paradigm. Nat Rev Genet 12: 565-575.

Gardner A (2014). Genomic imprinting and the units of adaptation. Heredity 113 104-111.

Gregg C, Zhang J, Weissbourd B, Luo S, Schroth GP, Haig D et al. (2010). High-resolution analysis of parent-of-origin allelic expression in the mouse brain. Science 329 . 643-648

Haig D (2014). Coadaptation and conflict, misconception and muddle, in the evolution of genomic imprinting. Heredity 113: 96-103.

Haig D, Graham C (1991). Genomic imprinting and the strange case of the insulin-like growth factor II receptor. Cell 64: 1045-1046

Hanna CW, Kelsey G (2014). The specification of imprints in mammals. Heredity (doi: 10.1038/hdy.2014.54).

Kelsey G, Bartolomei MS (2012). Imprinted genes . . . and the number is? PLoS Genet 8 : e1002601. 
Keverne EB (2014). Mammalian viviparity; a complex niche in the evolution of genomic imprinting. Heredity 113: 138-144.

Lachmann M, Blackstone NW, Haig D, Kowald A, Michod RE, SzathmÃry E et al. (2003). Group report: Cooperation and conflict in the evolution of genomes, cells, and multicellular organisms. in: (ed Hammerstein P) (Genetic and Cultural Evolution of Cooperation. MIT Press: Cambridge, Mass 327-356.

Lee JT, Bartolomei MS (2013). X-inactivation, imprinting, and long noncoding RNAs in health and disease. Cell 152: 1308-1323.

Metz CW (1938). Chromosome behavior, inheritance and sex determination in Sciara. Am Nat 72: 485-520.

Moore T, Haig D (1991). Genomic imprinting in mammalian development: a parental tugof-war. Trends Genet 7: 45-49.

Okae H, Hiura H, Nishida Y, Funayama R, Tanaka S, Chiba H et al. (2012). Reinvestigation and RNA sequencing-based identification of genes with placenta-specific imprinted expression. Hum Mol Genet 21: 548-558.

Patten MM, Ross L, Curley JP, Queller DC, Bonduriansky R, Wolf JB (2014). The evolution of genomic imprinting: theories, predictions, and empirical tests. Heredity 113: $119-128$
Reik W, Collick A, Norriss ML, Barton C, Surani MA (1987). Genomic imprinting determines methylation of parental alleles in transgenic mice. Nature 328: 248-251.

Sapienza C, Peterson AC, Rossant J, Balling R (1987). Degree of methylation of transgenes is dependent on gamete of origin. Nature 328: 251-254.

Smits G, Mungall AJ, Griffiths-Jones S, Smith P, Beury D, Matthews L et al. (2008). Conservation of the H19 noncoding RNA and H19-IGF2 imprinting mechanism in therians. Nat Genet 40: 971-976.

Spencer HG, Clark AG (2014). Non-conflict theories for the evolution of genomic imprinting. Heredity 113: 112-118.

Stringer JM, Pask AJ, Shaw G, Renfree MB (2014). Post-natal imprinting: evidence from marsupials. Heredity 113: 145-155.

Wang X, Clark AG (2014). Using next-generation RNA sequencing to identify imprinted genes. Heredity 113: 156-166.

Wolf JB, Brandvain Y (2014). Gene interactions in the evolution of genomic imprinting. Heredity 113: 129-137.

Wolf JB, Hager R (2006). A maternal-offspring coadaptation theory for the evolution of genomic imprinting. PLoS Biol 4: 2238-2243.

Wolf JB, Oakey RJ, Feil R (2014). Imprinted gene expression in hybrids: perturbed mechanisms and evolutionary implications. Heredity 113: 167-175. 